\title{
Dynamics of vorticity
}

\author{
By P. G. SAFFMAN \\ Applied Mathematics, California Institute of Technology, Pasadena, California 91125
}

Remarks are made about the status of research on the role of vorticity in fluid dynamics and some unsolved problems of current interest are described.

\section{Introduction}

Why is the vorticity, $\boldsymbol{\omega}=$ curl $\mathbf{u}$, so important? It is not easily measurable (the invention of an accurate, non-intrusive, fine-resolution vorticity meter would be of inestimable value for a number of experimental and theoretical applications) and appears from its definition to have no particular physical significance; nothing seems to distinguish it especially from other gradients of the velocity field. Yet, at least for the motion of homogeneous incompressible fluids, it is the property of the flow field of crucial import, and it is not an exaggeration to say that all the problems of such flows can be posed as questions about the strength and location of the vorticity. There are, as is well known, two principal reasons for this. Firstly, if $\omega=0$ everywhere in an incompressible fluid, then the fluid really ceases to be a fluid; it loses its infinite number of degrees of freedom, which make possible the infinite variety of fluid motion, and becomes a flexible extension of the bodies whose movement generates the flow; bring the walls to rest and the fluid stops immediately. (The last statement is not literally true if the region is multiply connected, but this is a detail.) The motion of solids through liquids in which $\omega \equiv 0$ can be reduced to a branch of classical mechanics and the effect of the fluid can be described completely in terms of a Lagrangian which depends only on the co-ordinates of the bodies (and certain cyclic constants if multiply connected). Lamb (1932, chap. VI) gives a thorough account of this branch of classical fluid mechanics, whose irrelevance to the real world is summarized by the D'Alembert paradox of zero drag in steady flow. The mathematical cause of the loss of fluidic properties is the uniqueness properties of Laplace's equation; the physical reason is the relation of vorticity to the local spin of the fluid and the constraints imposed by the absence of spin, e.g. an infinitesimal cube does not rotate. Secondly, the distribution of vorticity is often compact, even though the velocity and pressure fields extend everywhere, and remains so by virtue of the Helmholtz laws of vortex motion, apart from viscous diffusion. Thus the specification of the vorticity field may be far more economical than that of the velocity.

In a real fluid, the no-slip boundary condition or the singularities near sharp corners or non-conservative body forces (associated perhaps with density differences) produce vorticity which is convected or diffuses into the fluid. The regions containing vorticity may be idealized as surfaces of discontinuity (vortex sheets or boundary layers) or lines (point vortices) but in dealing with the motion of real fluids we must drop the condition $\boldsymbol{\omega} \equiv 0$. Then, to quote Lamb $(1932, \S 159 a)$ 'The motion of a solid in a liquid endowed with vorticity is a problem of considerable interest, but is unfortunately not very tractable.' Alternatively, ask any scientist what is the great unsolved problem of 
fluid dynamics and the immediate answer is turbulence. What is turbulence but a random chaotic field of vorticity, whose strong nonlinear interactions makes the problem so impossibly difficult?

Generations of scientists working in fluid dynamies have recognized the importance of vorticity. It has provided a powerful qualitative description for many of the important phenomena of fluid mechanies; e.g. the formation and separation of boundary layers have been so described in terms of the production, convection and diffusion of vorticity, the dissipation of energy at a rate practically independent of the viscosity in turbulent flows is explained by the amplification of vorticity by the stretching of vortex lines, the lift on a wing is explained by the bound vorticity and trailing vortex structure, and most recently the concept of the coherent structure in turbulent shear flows has led to the picture of such flows as a superposition of organized, 'deterministic' vortices whose evolution and interaction is the turbulence. On the other hand, the strong nonlinearity of the equations of vortex motion has made quantitative use of the concept difficult. Flow geometries which lend themselves to analytical investigation or simple numerical treatment have with few exceptions been of limited fluid-dynamical interest. But the computer is now changing the picture and giving the modern theoretical fluid-dynamicist a tool with a power far greater than those available to the great scientists who founded and developed the subject. Numerical computations are now possible which were just hopes 25 years ago when the Journal of Fluid Mechanics was founded. Some of these have been described in recent review articles (e.g. Saffman \& Baker 1979, Leonard 1980) and others are in press. Our modern ability to calculate vortex motions is also leading to the realization that almost irrotational motions such as surface waves on uniform irrotational fluid or not too small and not too large air bubbles in water may be successfully treated as problems of vortex motion, $\uparrow$ the free surface of water waves or the boundary of a bubble being a vortex sheet whose position satisfies an integro-differential equation. As Kelvin (1880) remarked in the context of an investigation on vortex motion, 'crowds of exceedingly interesting cases present themselves'. Rather than describe all these accomplishments, it is perhaps more suitable and in accord with the spirit of the present issue of this journal to discuss some of the outstanding problems of both mathematical and physical interest that are specifically in the area of vortex dynamies. Even with super-powerful computers like the Control Data Corporation CYBER 205 and the CRAY 1, and the increased understanding of the mathematical properties of nonlinear integro-differential equations, these problems tax to the utmost our capabilities, but it is expected that significant progress will be reported in papers that appear in the next 25 years of this journal.

\section{The inviscid Taylor-Green problem}

One of the unsolved mathematical problems of three-dimensional motion is the global existence of solutions of the initial-value problem for the Navier-Stokes equations. Perhaps even harder is the question of existence when viscosity is put identically equal to zero and the Euler equations are solved. In this case, it has been speculated for many years that the nonlinear stretching of vortex lines may proceed

$\dagger$ The second example was suggested by Professor D. W. Moore. 
at a rate faster than exponential and some lines may become infinitely long in a finite time, signifying a singularity in the vorticity field.

The existence problems in three dimensions are delicate and apparently outside the scope of present mathematical techniques. The vorticity equation in three dimensions can be written for inviscid incompressible flow as

$$
\frac{d \omega_{i}}{d t}=e_{i j} \omega_{j}
$$

where

$$
e_{i j}=\frac{1}{2}\left(\frac{\partial u_{i}}{\partial x_{j}}+\frac{\partial u_{j}}{\partial x_{i}}\right)
$$

is the rate-of-strain tensor, and $d / d t$ denotes differentiation following the fluid. If $e_{i j}$ at a point were proportional to the local vorticity, then (2.1) would certainly suggest strongly (and rigorous proofs should not be hard) that $d \omega / d t \propto c \omega^{2}$ and $\omega=\infty$ in a finite time $(\omega=|\omega|)$. The difficulty is that $e_{i j}$ at a point depends only on the irrotational part of the local velocity field and not directly on the local value of $\omega$. Thus the increase in vorticity following a fluid particle depends on the correlation between the vorticity of different elements of the fluid, and the global dependence of velocity on vorticity, expressed mathematically through the vector potential formulae

$$
\mathbf{u}=\operatorname{curl} \mathbf{A}, \quad \boldsymbol{\omega}=-\nabla^{2} \mathbf{A}, \quad \mathbf{A}=\frac{1}{4 \pi} \int \frac{\boldsymbol{\omega}^{\prime}}{r} d x^{\prime}
$$

has not yet allowed the construction of suitable a priori estimates.

Taylor \& Green (1937) attacked this problem for the Navier-Stokes equations by expressing the solution of an initial-value problem as a power series in $t$. The particular initial flow field chosen was

$$
u=\cos x \sin y \cos z, \quad v=-\sin x \cos y \cos z, \quad w=0,
$$

over all space. The periodicity is invariant and it is sufficient to restrict attention to a cube of side $2 \pi$ with periodic boundary conditions. They wanted to demonstrate the growth of vorticity for small viscosity, and calculating by hand they found the coefficient of $t^{4}$ in the Taylor series expansion of $\overline{\omega^{2}}$.

Recently, Morf, Orszag \& Frisch (1980) have extended this calculation, using the computer to do the arithmetic, and found the coefficients of terms up to $t^{44}$ for the inviscid case $\nu=0$. The odd powers vanish identically so 22 coefficients were actually found; the calculation of these coefficients and similar ones for the mean values of higher powers of the velocity field required $7 \mathrm{~h}$ on a CDC 7600 . The purpose of this calculation is to determine the singularities of $\overline{\omega^{2}}$ regarded as an analytic function of the complex variable $t$. Since the initial conditions are smooth, the function is analytic at $t=0$. The task is to continue the function analytically, given a finite number of its Taylor series coefficients at the origin, and find in particular if there are singularities on the positive real axis. If no more information is given, the problem is ill-posed, but the hope is that one can nevertheless estimate from a finite number of coefficients the positions and nature of the singularities by the methods of Domb-Sykes plots and Padé approximants, etc. This technique, originally developed in statistical mechanics, has had some success in fluid mechanies, especially in finite-amplitude gravity waves on deep water, but it is a 'black box' and the results can never be taken as more than suggestive until an independent verification is given. The interesting result of Morf 
et al. (1980) is the finding by these techniques of a branch point singularity with negative exponent at $t=t_{c} \doteqdot 5 \cdot 2$.

If this result is correct, and the speculation is confirmed that the initial-value problem for the Euler equations in three dimensions is in general ill-posed, it must rank as one of the more important discoveries of fluid mechanics. The possible appearance of a spontaneous singularity in three-dimensional motion would strike a blow at the validity of the myriad of inviscid two-dimensional unsteady calculations, all relying for applicability on the hope that three-dimensionality and viscosity are small perturbations, since it would imply that viscosity is always effective in a finite time independent of its value when the flow is three-dimensional. It would also, for example, provide justification for the assumption of an inviscid energy cascade in high Reynolds number turbulence. On a different level, it would demonstrate conclusively the role of the computer as a major weapon for the qualitative investigation and discovery of phenomena, on a par with experiment and classical analysis, and more productive so far in any real sense than the techniques of 'modern mathematics'. But rather more convincing evidence than the Morf et al. (1980) calculation is needed before the result can be accepted with confidence. This problem is one of the most challenging of the present time for both the mathematician and the numerical analyst.

It is appropriate to mention here a related problem, physically less important but mathematically and computationally just as interesting, namely the existence of unsteady inviscid vortex sheets in two dimensions. Three-dimensional vortex sheets would just constitute a special case of the three-dimensional Euler equation, but in two dimensions it is established that solutions of the Euler equations exist for all time if the initial data is smooth. The vortex sheet is not a smooth solution, but it is one which is used to model important physical phenomena, such as the Kelvin-Helmholtz instability of shear layers and the formation of wakes by the roll-up of separating boundary layers. If, in general, a two-dimensional vortex sheet develops a singularity in a finite time, the consequences for our analysis of many flows would be nonnegligible. Recently, Moore (1979) has presented suggestive evidence that vortex sheets of zero thickness do indeed develop singularities in a finite time, caused by the nonlinear excitation of short waves which amplify rapidly. The physical mechanism is fundamentally different from that which causes the claimed three-dimensional singularity, since there is no vortex line stretching in two dimensions, and can perhaps be thought of as a result of unbalanced pressure forces over highly crinkled vortex sheets. It can also be expected to apply in three dimensions, after vortex stretching produces sheets of intense vorticity, but whether the two mechanisms interfere constructively or destructively remains to be elucidated by analysis and computation.

\section{Inviscid flow past bodies}

Another of the challenging unsolved problems of fluid mechanics is the limiting form of steady solutions of the Navier-Stokes equations describing flow past bluff bodies as $\nu \rightarrow 0$. This problem is often dismissed as unrealistic and of academic interest only, since in reality the boundary layer and/or wake become turbulent. But there always exists the possibility that a better understanding of the fluid mechanies coupled to feed-back control or changes of geometry may enable the suppression of the instabilities 
or at least delay their onset, allowing the advantages of laminar flow to be exploited. The contribution of vortex dynamies to the problem lies in the determination of the possible inviscid but not necessarily irrotational flow states.

The irrotational flow past a body is unique, except for some arbitrariness associated with multiple connectivity. But there exist an infinite number of rotational flows depending upon the allowed vorticity distributions. Three special cases seem to be of most interest as most likely to be realizable or worth considering as the $v=0$ limit of Navier-Stokes solutions. One point should be made clear; there is no reason why the boundary conditions should not be functions of $\nu$, e.g. there could be blowing or suction through the boundary at rates depending on $\nu$, and the limit as $\nu \rightarrow 0$ need not be unique. Indeed, the continual discovery of new bifurcation phenomena in fluid mechanics would make it surprising if the limit were unique. Professor Roshko has commented that flow past a thick airfoil is a possible example, for which plausible alternative motions could be either a flow separating near the section of maximum thickness with the tail in the dead-water region of the near wake or the streamline irrotational flow around the entire body.

The first case (i) is the Kirchhoff-Helmholtz limit, in which vortex sheets emanating from the body and stretching to infinity separate a region of stagnant fluid from the irrotational stream. There is some arbitrariness here in determining the point of separation but it can usually be handled by a minimum singularity type hypothesis. This case has the advantage that it predicts a finite drag and that in two dimensions exact solutions can be calculated for polygonal bodies.

The second ease (ii) will be designated the attached vortex model. Here, finite vortices are supposed attached to the body and the flow at infinity is irrotational. The vorticity is finite so that the velocity is continuous, i.e. the edges of the vortices are not vortex sheets. The Helmholtz laws for steady motion impose some constraints on the vorticity distribution, e.g. in two dimensions the vorticity must be a function of the stream function, but there will be a large amount of arbitrariness in the size and structure of the vortices. A degenerate example of a flow of this kind is Föppl's calculation of the flow past a circular cylinder with two point vortices standing in the wake (see Lamb 1932, § 155). A similar example is the Saffman \& Sheffield (1977) calculation of flow past an inclined flat plate with a single vortex standing over the plate, which showed the possibility of considerable lift enhancement if such a flow could be generated. Solutions with finite vortices have not yet been computed. This case suffers from the disadvantage (?) of predicting zero drag. A more serious objection is that separating streamlines leave perpendicularly (at least in two dimensions) and fitting viscous boundary layers might be impossible, even with surface suction, so these flows would not be $\nu=0$ limits.

Case (iii), the Batchelor (1956) limit, is the attached vortex flow with vortex sheets bounding the outside edges of the vortices and uniform vorticity inside each one in two dimensions, and the appropriate modification for axisymmetric flow. This fow can be regarded as a hybrid of the first two cases; the advantage of allowing vortex sheets is that the streamlines can presumably separate smoothly, increasing the chance of these flows being $\nu=0$ limits. It appears, however, that Batchelor flows have not yet been computed so that their existence remains an open question.

It should perhaps be stressed that existence of vortex flows is a non-trivial question, not just a mathematical nicety or an exercise in proving the obvious. Recent calcula- 

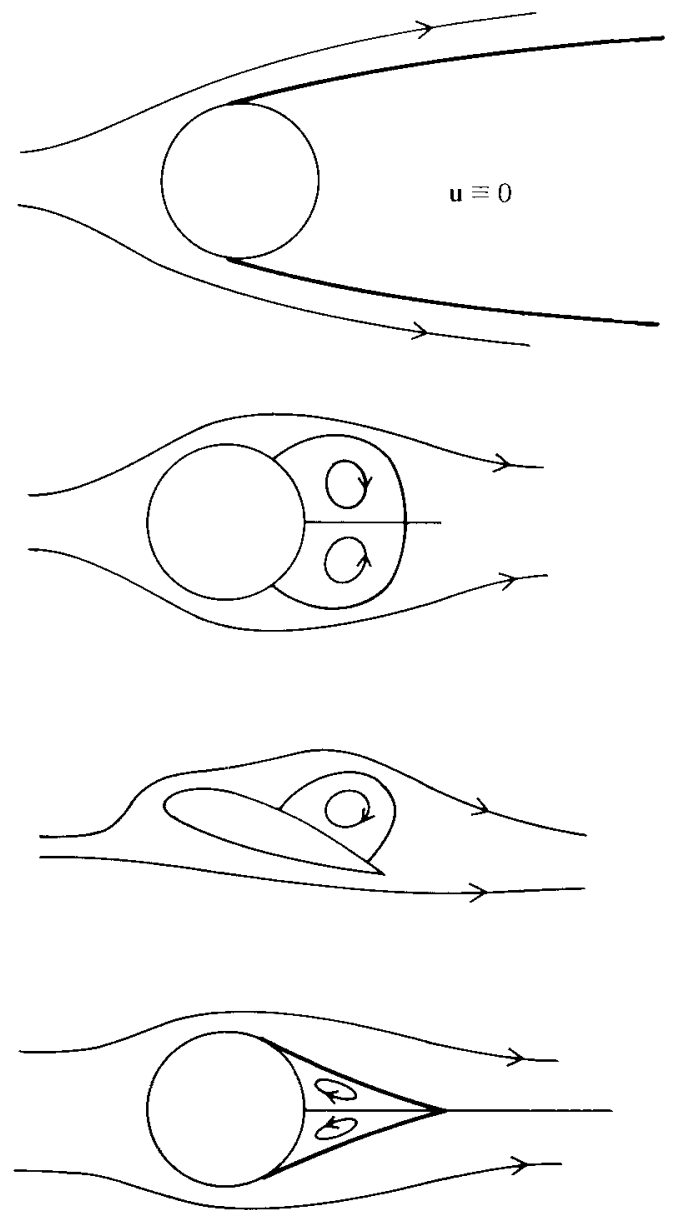

(iii)

Frgure 1. Sketches of possible inviscid rotational flow past a bluff body. (i) KirchhoffHelmholtz flow. Vortex sheets bound an infinite stagnant region. Finite drag. (ii) Flows with attached vortices. No vortex sheets. $a$, symmetrical flow past a bluff body; $b$, unsymmetrical flow past a wing. Zero drag but lift in case $b$. (iii) Batchelor flow. Vortex sheets bound attached vortices. Zero drag.

tions of steady two-dimensional arrays of finite vortices (e.g. Saffman \& Szeto 1981) have shown that there are no solutions if the vortices are too large, so existence of solutions cannot just be taken for granted.

The three cases are sketched in figure 1. For two-dimensional flow and constant vorticity, integro-differential equations can be derived for the unknown shapes of the vortex sheets or bounding streamlines. It is nowadays a reasonably straightforward matter to obtain solutions of the equations using global Newton methods, provided a sufficiently large computer and adequate computing time are available. The principal difficulty lies in ensuring that the solution of the discretized system actually approximates a solution of the integro-differential equation since nothing more than consistency checks seem to be available.

Once the inviscid rotational flows have been catalogued, we are of course faced with the yet more difficult task of finding which are realizable. Perhaps this problem will be 
discussed in the issue commemorating the 50th anniversary of the Journal of Fluid Mechanics.

\section{Vortex stability and interactions}

The stability of steady vortex configurations in an inviscid fluid to infinitesimal and finite-amplitude disturbances is of considerable interest but poses great difficulties. The Kelvin-Helmholtz instability of vortex sheets has been thoroughly examined, as has also the stability of steady configurations of point vortices to two-dimensional disturbances motivated strongly by interest in the Kármán vortex wake behind bluff bodies and more recently by studies of Helium II. But there are still puzzles here, and a large number of other challenging and important problems exist for which ignorance reigns supreme.

The Kármán vortex street of two infinite staggered rows of opposite signed vorticity is a particular mystery. Observations of vortex wakes, for which the street is a model, suggest that the street is stable, yet calculations show it is unstable. The particular spacing ratio 0.281 found by Kármán to give neutral stability is now known to be spurious; it is a momentary vanishing of the growth rate of infinitesimal disturbances due to a coincidence of eigenvalues and the configuration is unstable to finite-amplitude disturbances. The common speculation is that giving the vortices finite size will stabilize the array to two-dimensional disturbances, and there is some numerical (Christiansen \& Zabusky 1973) and analytical (Saffman \& Schatzman 1980) evidence to support this belief, but completely convincing evidence is lacking. As regards the stability to three-dimensional disturbances, there are a couple of 50-year-old incomplete investigations of stability to long wave axial disturbances, but otherwise nothing.

Indeed the whole question of three-dimensional stability is wide open. (The Squire theorem for parallel shear layers has perhaps had an inhibiting effect on stability calculations.) There is probably a wide range of phenomena waiting to be discovered. As an example, see the results described by McLean et al. (1980) on the stability of finite-amplitude water waves, which can be regarded as non-uniform vortex sheets, to three-dimensional disturbances.

A particular question worthy of study is the difference in the stability of inviscid flows of boundary-layer type, wake type, or free shear layer type to three-dimensional disturbances. In the first instance, each flow can be modelled by a vortex sheet of finite thickness; the type of flow corresponds to either the presence of a rigid wall or the requirement of symmetry or antisymmetry. It is expected that these flows will have a primary instability or bifurcation into a steady non-uniform flow. Two-dimensional finite-amplitude non-uniform flows have been calculated for the free shear layer (Stuart 1967, Saffman \& Szeto 1981), and there is no obvious reason why they should not also exist for the boundary-layer type configuration or with the staggered symmetry appropriate for a wake. In fact, there is no obvious reason why three-dimensional, finite-amplitude steady flows should not exist for each case. The interesting question now is the secondary instability of these configurations to arbitrary threedimensional disturbances. The experimental evidence suggests as a plausible speculation that the free shear layer type is weakly unstable, the wake type is moderately unstable, and the boundary-layer type is strongly unstable, but it remains to be seen if this is confirmed by calculation. 


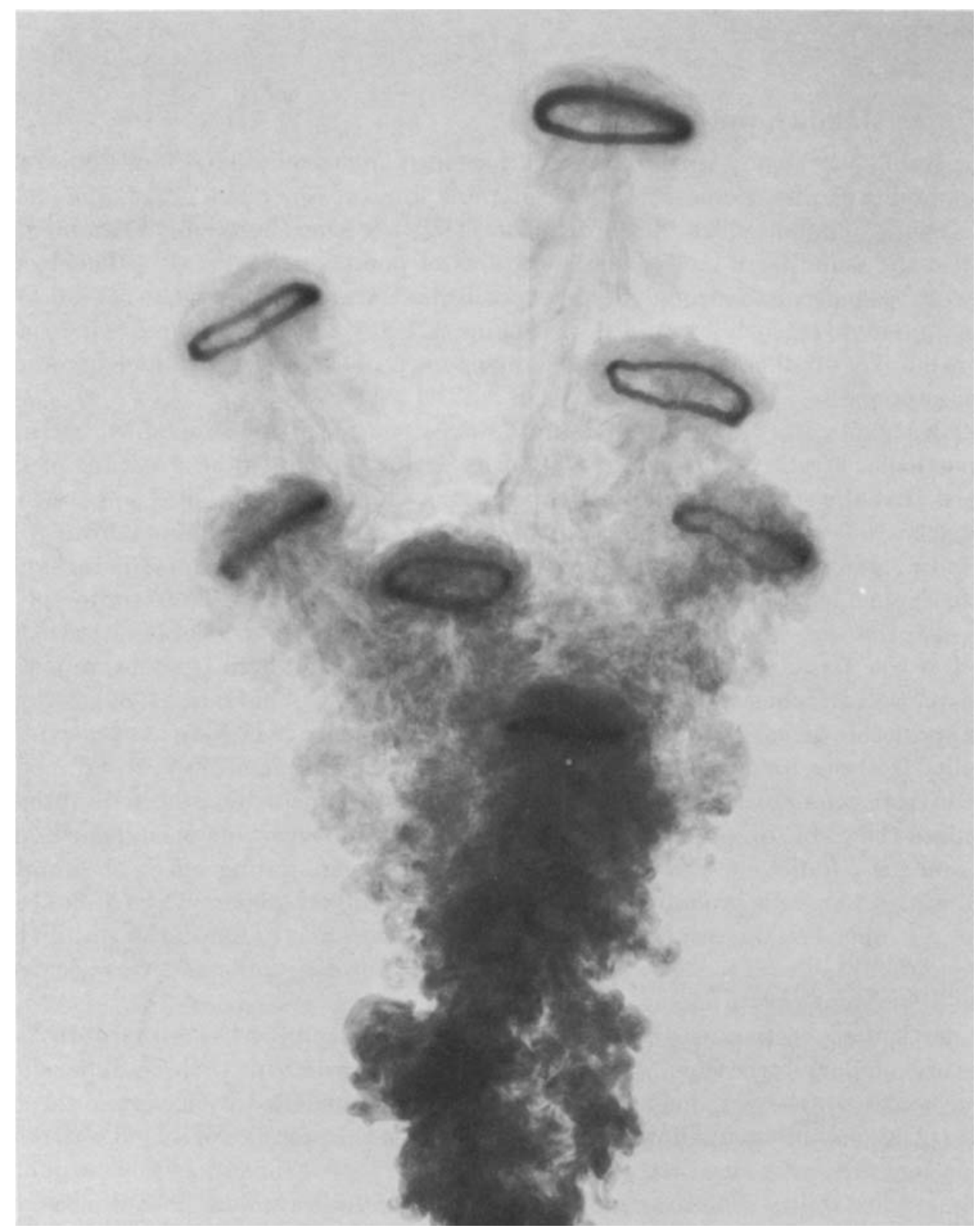

Figure 2. Photograph of vortex interactions by Glezer (1981). Eight vortex rings formed from pulses of $20 \mathrm{~ms}$ duration are shot one after the other at $85 \mathrm{~ms}$ intervals and separate after going about 20 diameters in a spectacular blooming. The leading vortex ring is out of the picture.

The computing resources now exist to find the eigenvalues of systems governed by partial differential equations. The ability to separate variables and reduce to ordinary differential equation eigenvalue problems is no longer essential. The stability of nonparallel flows to two- and three-dimensional infinitesimal disturbances is then a feasible calculation; the only technical limitation would seem to be the availability of the computer resources. It can be argued that we are entering a stage of research in which 
the problem is to decide what to caloulate, not how to calculate. Strangely enough, this will make analysis more, not less, important because of the needs first to provide checks on the flood of numbers and second to guide, organize, interpret and make sense of the computations. Rather than study infinitesimal disturbances and calculate spectra, some investigators who have developed unsteady codes study instability by calculating initial-value problems. There is the advantage here that finite-amplitude effects are automatically taken into account, but there is the serious drawback that only a few cases can be considered and a complete picture cannot be obtained. The extent to which the initial excitation is typical is always arguable. Also sound methods need to be developed to distinguish between numerical instability and transition to turbulence; all too often the former is identified as the latter. In three-dimensional inviscid calculations, the ill-posedness discussed in $\$ 2$ may be a factor.

One particular motion exemplifies the whole range of problems of vortex motion and is also a commonly known phenomenon, namely the vortex ring or smoke ring. Vortex rings are easily produced by dropping drops of one liquid into another, or by puffing fluid out of a hole, or by exhaling smoke if one has the skill. Their formation is a problem of vortex sheet dynamics, the steady state is a problem of existence, their duration is a problem of stability, and if there are several we have the problem of vortex interactions. Saffman \& Baker (1979) in their review of vortex interactions commented that they knew of no well-documented case of vortex ring leapfrogging; in full accordance with Murphy's law or one of its corollaries, two papers showing this were found after the article was in press (Oshima 1978, Yamada \& Matsui 1978).

One of the most fascinating examples of vortex interactions is provided by the collision of vortex rings. Under the appropriate circumstances, two rings shot at one another in a plane will merge into a single, ellipse-like ring, which oscillates and then breaks up into two separate rings moving in a plane normal to the original one. Each new ring is a mixture of the old ones. Oshima \& Asaka (1975) show colour photographs of a red and yellow ring colliding and separating after collision into two rings each half yellow and half red. The details of the breaking and rejoining of vortex lines which takes place when the vortices collide and reform is a mystery awaiting satisfactory explanation.

Figure 2 is a remarkable photograph by Glezer (1981) of vortex ring interaction. Numbers of vortex rings are shot from an orifice one after another at short (typically $50-100 \mathrm{~ms}$ ) intervals. For some distance they travel along a common axis, and then separate in a spectacular manner. This phenomenon is fairly repeatable and is an apt demonstration of the complexities of vortex motion.

\section{Conclusion}

There can be no doubt that in any list of the most important papers in fluid mechanies, a prominent place will be held by Helmholtz's great paper 'Über Integrale der hydrodynamischen Gleichungen welche den Wirbelbewegungen entsprechen' $\dagger$ whose appearance in 1858 laid the foundations of the study of rotational or 'vortex' motion and together with the subsequent papers of Lord Kelvin provides the basis

† For an English translation, see Helmholtz (1867). The paper contains an extract from a letter by Lord Kelvin to P. G. Tait, in which he states without proof the classic formula for the speed of a thin vortex ring. This formula has caused much anguish. 
for the understanding and description of rotational fluid motion under conditions such that the direct effect of viscosity is not important. A measure of the quality and significance of the paper is that, although it is 123 years old, it is as good and clear an exposition as any, and is old-fashioned only in the use of Cartesian notation (like Lamb's 1932 text) rather than the modern vector or tensor notation. It is not surprising that Lord Kelvin (1867) was so moved by the elegant concept of vorticity and vortex rings in a perfect fluid to propose his theory of vortex atoms. Although the application to the structure of matter was short lived, the study of the dynamics of vorticity has remained active in fluid mechanics research. The computer is giving the theoretician a new and potent tool which can be expected to have powerful consequences, and the experimental discoveries of coherent structures in turbulence are providing a further practical impetus for the investigation.

\section{REFERENCES}

BATCHELOR, G. K. 1956 A proposal concerning laminar wakes behind bluff bodies at large Reynolds number. $J$. Fluid Mech. 1, 388-398.

Christransen, J. P. \& Zabusky, N. J. 1973 Instability, coalescence and fission of finite-area vortex structures. $J$. Fluid Mech. 61, 219-243.

GLezer, A. 1981 Ph.D. thesis, California Institute of Technology.

Helmoutz, H. 1867 On integrals of the hydrodynamical equations which express vortex motion. Phil. Mag. 33, 485-512.

Kelvin, Lord 1867 On vortex atoms. Phil. Mag. 34, 15-24.

Kelvin, LoRd 1880 Vibrations of a columnar vortex. Phil. Mag. 10, 155-168.

LAMB, H. 1932 Hydrodynamics. Cambridge University Press.

Leonard, A. 1980 Vortex methods for flow simulation. J. Comput. Phys. (to appear).

Mclean, J. W., Ma, Y. C., Martin, D. U., Saffman, P. G. \& Yuen, H. C. 1980 Three-dimensional instability of finite amplitude gravity waves. Phys. Rev. Lett. (sub judice).

Morf, R. H., Orszag, S. A. \& Frisch, U. 1980 Spontaneous singularity in three-dimensional, inviscid incompressible flow. Phys. Rev. Lett. 44, 572.

Moore, D. W. 1979 The spontaneous appearance of a singularity in the shape of an evolving vortex sheet. Proc. Roy. Soc. A 365, 105-119.

Oshima, Y. 1978 The game of passing through of a pair of vortex rings. J. Phys. Soc. Japan 45, $660-664$.

Oshima, Y. \& ASAKA, S, 1975 Interaction of two vortex rings moving side by side. Nat. Sci. Rep. Ochanomizu Univ. 26, 31-37.

Saffman, P. G. \& Baker, G. R. 1979 Vortex interactions. Ann. Rev. Fluid Mech. 11, 95-122.

Saffman, P. G. \& Schatzman, J. C. 1980 Properties of a vortex street of finite vortices. S.I.A.M. In. Sci. Stat. Computing (sub judice).

Saffman, P. G. \& Sheffield, J. S. 1977 Flow over a wing with an attached free vortex. Stud. Appl. Math. 57, 107-117.

Saffian, P. G. \& Szeto, R. 1981 Structure of a linear array of uniform vortices. Stud. Appl. Math. (to appear).

StUaRT, J. T. 1967 On finite amplitude oscillations in laminar mixing layers. J. Fluid Mech. 29, 417-440.

Taylor, G. I. \& GReen, A. E. 1937 Mechanism of the production of small eddies from large ones. Proc. Roy. Soc. A 158, 499-521.

Yamada, H. \& MATSUi, T. 1978 Preliminary study of mutual slip-through of a pair of vortices. Phys. Fluids 21, 292-294. 